\title{
Bronchodilator treatment for partially reversible chronic obstructive airways disease.
}

\author{
M K Tandon, S G Kailis
}

\begin{abstract}
The effect of six weeks' treatment with inhaled terbutaline (1 $\mathrm{mg}$ four times a day), optimised doses of theophylline (twice a day), the combination of theophylline and terbutaline, and placebo was studied in a randomised, double blind, crossover trial. Thirty patients with partially reversible chronic airflow obstruction and a mean forced expiratory volume in one second $\left(F E V_{1}\right)$ of 1.2 litres that improved by $25 \%$ were included in the study. Patients who developed non-infective exacerbations of airflow obstruction that required additional bronchodilator treatment were classed as "treatment failures." Such treatment failure occurred in 23 patients with placebo, in 22 patients with theophylline, in 12 patients with terbutaline, and in two patients taking the two drugs. Mean daily peak flow readings were highest with the combination of the two drugs, followed by terbutaline and then theophylline, and lowest with placebo. Thus a combination of terbutaline and theophylline was superior to either drug alone; inhaled terbutaline was superior to theophylline alone. Theophylline alone does not appear to have much place in the management of patients with partially reversible obstructive airways disease.
\end{abstract}

Though some studies on combination bronchodilator treatment have shown that at least an additive effect is achieved when a beta ${ }_{2}$ agonist and a methylxanthine are given together, ${ }^{12}$ there is still controversy about this. In patients with chronic obstructive airways disease in one study treament with inhaled metaproterenol for one week produced significant improvement in spirometric values, exercise tolerance, and subjective symptoms, whereas treatment with theophylline did not. ${ }^{3}$ There was no benefit from combining the two drugs.

Dull and Alexander reviewed various studies of treatment with theophylline in patients with chronic obstructive airways disease and concluded that theophylline does not improve symptoms. ${ }^{4}$ Others have shown that older patients with chronic obstructive airways disease tolerate theophylline poorly, commonly complaining of insomnia and irritability, and they suggest that theophylline may even be harmful. ${ }^{5}$ Elderly patients may have impaired elimination of theophylline ${ }^{6}$ and hence be more likely to develop side effects. Guyatt et $a l^{7}$ studied the effects of oral theophylline, inhaled salbutamol, and the combination on airway function, exercise capacity, and quality of life, in patients with chronic obstructive airways disease. They observed similar improvement with the two drugs and additional benefit when the drugs were combined. When Taylor et $a l^{8}$ studied oral theophylline, inhaled salbutamol, and a combination of the two in patients with chronic obstructive airways disease over three weeks they found that "treatment failures" (non-infective exacerbations of airflow obstruction) were fewest with combined treatment and most with salbutamol, theophylline being intermediate.

In view of the controversy about the relative efficacies of oral theophylline, inhaled beta $_{2}$ agonists, and their combinations we carried out a randomised, double blind, placebo controlled crossover study in patients with partially reversible chronic obstructive airways disease to assess the relative efficacies of optimal doses of oral theophylline, inhaled terbutaline, a combination of the two drugs, and placebo in keeping them symptom free and improving in lung function for the six weeks of each regimen.

\section{Patients}

Thirty seven men, aged 55-85 years, attending the chest clinic at Repatriation General Hospital for regular review of their chronic obstructive airways disease were included in the study. Ten patients were smokers and 27 ex-smokers. The criteria for inclusion in the study were $(a)$ best postbronchodilator value for $\mathrm{FEV}_{1}$ in the past two years less than $65 \%$ of predicted normal; $(b)$ an improvement of over $15 \%$ in $\mathrm{FEV}_{1}$ after a bronchodilator aerosol on two previous occasions in the past six months; and (c) the need for regular bronchodilator treatment for optimal symptom control. On entry into the study all patients were taking inhaled beclomethasone and beta ${ }_{2}$ agonists; 34 patients were taking ipratropium bromide (withdrawn when they entered the study), 28 theophylline, and six oral corticosteroids.

Of the 37 patients, seven did not complete the study. Three were unwilling to comply with the study requirement to receive different combinations of drugs, two withdrew because of nausea and excessive flatulence while receiving theophylline, one developed a respiratory infection, and one was lost to follow up.

In the 30 patients who completed the study 
mean (SD) $\mathrm{FEV}_{1}$ at entry was $1 \cdot 22(0 \cdot 42)$ and mean vital capacity (VC) $2 \cdot 26(0 \cdot 48)$; the mean percentage improvement in $\mathrm{FEV}_{1}$ after $5 \mathrm{mg}$ nebulised terbutaline was $25 \cdot 4(12 \cdot 6)$. At this time all were in a stable clinical state. No patient with appreciable hepatic, renal, or cardiac dysfunction was included in the study.

DOSE TITRATION PERIOD

All patients had received treatment with theophylline previously or were currently receiving it and all had been able to tolerate treatment with it. Those patients receiving theophylline on inclusion into the study were asked to stop taking it for three days. All patients then entered a preparatory period during which the dose of oral theophylline was openly titrated to achieve a steady state peak serum theophylline level of $12-20 \mathrm{mg} /$ litre between five and six hours after ingestion of sustained release theophylline. All patients were capable of inhaling properly from a metered dose aerosol. During this preparatory period, the patients practised measuring and recording their peak expiratory flow rate, and charting medication intake on a daily record card.

\section{PROTOCOL}

After the preparatory period there was a two week washout. Patients were then allocated to receive, in a double blind manner, a sequence of four consecutive treatments, each for a period of six weeks, according to $4 \times 4$ Latin square design. During each treatment period the patient took four puffs of a metered dose inhaler four times daily at $0800,1400,1800$, and 2200 hours and individualised tablets twice daily at 0800 and 1800 hours. The four treatments consisted of the following: (1) Placebo tablets plus $1 \mathrm{mg}$ inhaled terbutaline from a metered dose canister $(250 \mu \mathrm{g} /$ puff $)$ fitted with a tube spacer of $150 \mathrm{ml}$ volume (Bricanyl Misthaler), four times daily; (2) sustained release theophylline tablets (Theodur), twice daily, and placebo for terbutaline aerosol with Misthaler; (3) sustained release theophylline tablets, twice daily, plus $1 \mathrm{mg}$ terbutaline aerosol, four times daily; (4) placebo tablets plus placebo inhaler.

For the four daily terbutaline or placebo inhalations the patients were instructed first to exhale to residual volume, then to activate the device, and then inhale to total lung capacity with breath holding for five seconds after each inhalation. The dose of theophylline was the one determined for that subject during the perparatory period to produce a steady state theophylline concentration in the range $12-20 \mathrm{mg} / 1$ 5-6 hours after administration.

Patients receiving treatment with inhaled beclomethasone or oral corticosteroids were allowed to continue treatment with these drugs at a constant dosage throughout the study; ipratropium bromide was not allowed.

$\mathrm{FEV}_{1}$ and vital capacity (VC) were measured at the start of the study and at the end of each treatment period as the best of three consecutive forced expirations on a 10 litre water filled spirometer (Expirograph Godard, Gould Instruments, Cleveland, Ohio). Before the baseline studies oral theophylline was witheld for 72 hours and inhaled beta ${ }_{2}$ agonists for six hours. At subsequent clinic visits patients were seen betwen 1300 and 1400 hours, when blood for serum theophylline assays was taken five to six hours after administration of the study medications in the morning. FEV $\mathrm{F}_{1}$ and $\mathrm{VC}$ were measured before and 30 minutes after administration of the study inhaler in the laboratory under supervision of laboratory staff. Plasma theophylline concentrations had either to be similar to those established during the preparatory treatment with oral theophylline or to be zero; if they were not, the patient was requested to take the prescribed treatment for another five days and serum theophylline concentrations were rechecked to ensure compliance with treatment. Slow release theophylline tablets were given in similar doses night and morning. The investigator (MKT) was unaware of the serum theophylline concentrations.

Daily measurements of peak expiratory flow (PEF) were obtained throughout the study 15 minutes after the drug inhalation at 0800 hours and at 2200 hours, a mini-Wright peak flow meter (Airmed, Clement Clarke Ltd, London) being used. The best of three readings was recorded on a diary card.

If a patient thought that he required additional bronchodilators, between the prescribed times, because of shortness of breath with wheeze, he was advised to contact the investigator (MKT). The patient was then allowed to use a separate salbutamol inhaler for symptom control. Salbutamol was allowed only from the onset of increased dyspnoea-that is, of treatment failure-to the morning of assessment. This period never exceeded 24 hours and no patient required more than 16 puffs of salbutamol. The patients were assessed within 24 hours of this contact and if this additional treatment was considered necessary because of a non-infective exacerbation of airflow obstruction the treatment regimen was deemed a "treatment failure." When a patient developed an exacerbation of airflow obstruction due to a chest infection, the study was suspended until he recovered, and resumed two weeks after recovery. We emphasised that all patients should continue to take the study medications as prescribed until they were assessed. On the day of assessment additional treatment with salbutamol was not permitted, so that the patient's lung function better reflected the response to the allocated treatment. The $\mathrm{FEV}_{1}$ and $\mathrm{VC}$ data reported are those obtained at the end of six weeks of treatment when this was completed without failure, or values obtained at the time of onset of failure when this was less than six weeks. Treatment with the next combination of drugs was then started after six weeks (a shorter period when treatment failure occurred).

The protocol was approved by the ethics committee of the Repatriation General Hospital and written consent for the study was obtained from each patient before entry into the study. 
ANALYSIS

Two way analysis of variance and paired $t$ tests were used to compare PEF data and paired $t$ tests with two tailed probability to compare $\mathrm{FEV}_{1}$ and $\mathrm{VC}$ values at the end of the four treatment regimens. Mean peak flow values for the whole of the study period were obtained. The numbers of treatment failures with different treatment regimens were compared by using Pearson's $\chi^{2}$ test. The F statistic was used to test for treatment-period interaction.

\section{Results}

The mean (SD) age of the 30 patients who completed the study was $70(4 \cdot 8)$ years. The daily dose of theophylline ranged from 400 to $1200 \mathrm{mg} /$ day. The mean serum theophylline concentration at the end of the preparatory period five to six hours after ingestion of tablets was $14.02(1.26)$ (range 11.5 to 17.4$) \mathrm{mg} / \mathrm{l}$.

\section{TREATMENT FAILURES}

The number of "treatment failures" during treatment with placebo and theophylline did not differ (table). There were fewer failures during treatment with inhaled terbutaline than during treatment with placebo or theophylline alone. Treatment with terbutaline and theophylline combined was significantly better in preventing treatment failure than either drug alone or placebo.

\section{PEF}

Mean values of morning and evening PEF during the four treatment periods 15 minutes after the study medications were higher during all active treatments than during placebo treatment (table), being highest with the two drugs combined, followed by terbutaline alone, theophylline alone being the worst of the active treatments $(p<0.01)$.

FEV $_{1}$ AND VC

The mean $\mathrm{FEV}_{1}$ during treatment with the two drugs combined was significantly higher than that during treatment with either drug alone or placebo (table). There was no significant difference in the $\mathrm{FEV}_{1}$ during treatment with theophylline alone and terbutaline alone.

Peak expiratory flow (PEF) and forced expiratory volume in one second $\left(F E V_{1}\right)$ during the four six week treatment periods in 30 patients

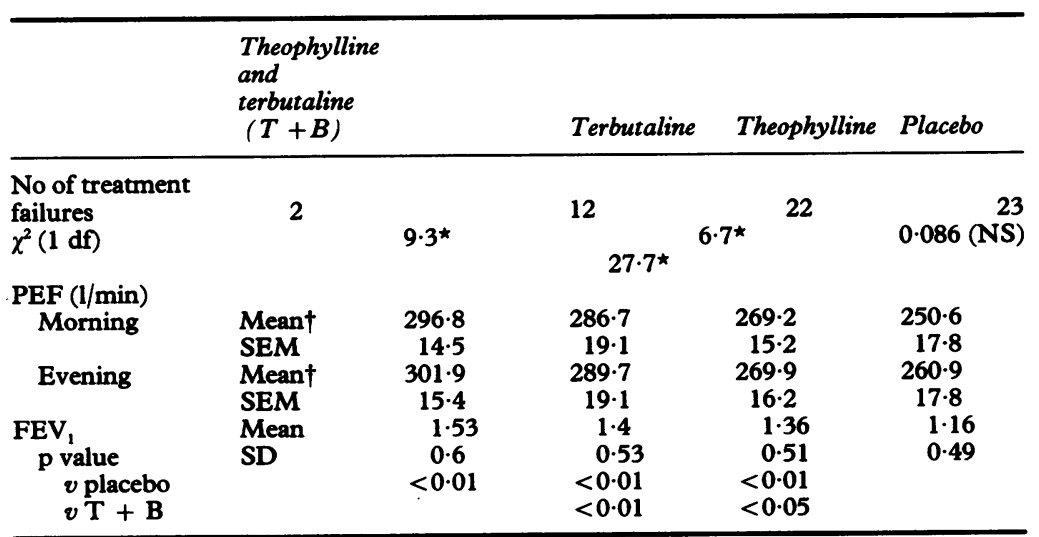

${ }^{\star} \mathrm{p}<0.01$.

$\dagger$ Mean readings for the duration of the treatments.
$\mathrm{FEV}_{1}$ values during treatment with theophylline and terbutaline alone were significantly higher than those observed after treatment with placebo. There was no significant difference in mean VC between the combined treatment and terbutaline alone; otherwise the results for $\mathrm{VC}$ were similar to those for $\mathrm{FEV}_{1}$. *

No treatment-period interaction was found for $\mathrm{FEV}_{1}$ or $\mathrm{VC}$ (for $\mathrm{FEV}_{1} \mathrm{p}=0.39$; for VC $\mathrm{p}=0 \cdot 71$ ).

\section{SIDE EFFECTS}

While receiving treatment with theophylline eight patients complained of gastrointestinal side effects. These symptoms were associated with a rise in serum theophylline concentration (mean $17 \cdot 7$ (range $16 \cdot 1-19 \cdot 4$ ) $\mathrm{mg} / \mathrm{l}$ ) from the mean run in concentration of 13.4 (range $12 \cdot 1-$ $15 \cdot 8) \mathrm{mg} / \mathrm{l}$. These patients required a reduction in the dose of theophylline.

\section{Discussion}

The results of this study show that in patients with partially reversible chronic obstructive airways disease treatment with a combination of oral theophylline and inhaled terbutaline was most effective in keeping the patient symptom free. Inhaled terbutaline was effective in $60 \%$ of patients whereas oral theophylline alone was not superior to placebo in preventing "treatment failure" as defined by the need for additional inhaled beta ${ }_{2}$ agonist drugs. The concept of "treatment failure" as the most important end point in assessing the efficacy of different regimens was used in this study because, if a particular form of treatment is to be clinically acceptable, it shoud be effective enough to keep patients free of exacerbations of airflow obstruction apart from those due to respiratory infection.

In this study patients received four puffs $(1.0 \mathrm{mg})$ of terbutaline daily, using a spacer device to improve drug delivery-a dose equivalent to more than the two puffs of salbutamol used by previous workers. ${ }^{78}$ In patients with severe airflow obstruction two puffs from a metered aerosol may not produce a response on the plateau of the dose-response

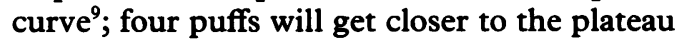
than two puffs. With this higher dose of terbutaline and a spacing device we were able to show the superiority of inhaled terbutaline over oral theophylline in terms of clinical response and mean daily peak flow readings. These results are in keeping with the findings of Dullinger et $a l^{3}{ }^{3}$ who found that inhaled metaproteronol was superior to theophylline. Dull et $a l^{10}$ suggested that a response to inhaled bronchodilator in the lung function laboratory may identify those patients who will respond to treatment with theophylline. Results of our study do not support their observation because, even though the patients included in this study had shown over $15 \%$ improvement in $\mathrm{FEV}_{1}$ after inhaling terbutaline before being included in the study, theophylline was ineffective in

* More detailed statistical analysis is available from the authors on request. 
preventing "treatment failures" in most of our patients.

In most studies looking at the effect of bronchodilators in patients with chronic obstructive airways disease the emphasis is on the effect of the drug on lung function during the study. We believe this is not the most appropriate measurement because in our study, though there were no significant differences in $\mathrm{FEV}_{1}$ or VC between treatment with terbutaline and with theophylline, there were significant differences in the number of treatment failures between the two drugs. Our results suggest that the effects of bronchodilators should be assessed from daily peak flow readings and adequacy of symptom control without additional bronchodilator treatment. These observations are in keeping with the findings of Anderson et al ${ }^{11}$ who failed to find any improvement of symptoms with theophylline despite a significant improvement in lung function.

Our results showing that the combination of inhaled beta ${ }_{2}$ agonist drugs and oral theophylline is superior to either drug alone are in keeping with findings of Guyatt et $a l^{7}$ and Taylor $e t a l^{8}$ and do not support the observations of Dullinger et al, ${ }^{3}$ who could not find any added benefit from combined drug treatment. Our finding that inhaled terbutaline is superior to theophylline is in keeping with the results of Dullinger $e t \mathrm{al}^{3}$ and does not support observations of Taylor et al,$^{8}$ who found theophylline to be superior to salbutamol. Guyatt et $a l^{7}$ found no difference between theophylline and salbutamol.

Our study suggests that for patients with partially reversible chronic obstructive airways disease treament with theophylline alone is not justified because of the high incidence of treatment failure. We recommend that treatment should initially be given with an inhaled sympathomimetic agent in a dose higher than is currently recommended. If an adequate response is not achieved additional treatment with theophylline should be tried to determine whether the desired improvement can be achieved.

We are grateful to Mr S Hare and Mr S Garg for performing lung function testing; to Professor D McNeil and Dr A Wood for statistical analysis of the results; and to Mr R Linden and Mr M Formato for carying out some of the theophylline assays. The study was supported by Astra Pharmaceuticals, North Ryde, New South Wales.

1 Shenfield GM. Combination bronchodilator therapy. Drugs 1982;24:414-39.

2 Smith JA, Weber RW, Nelson HS. Theophylline and aerosolized terbutaline in the treatment of bronchialasthma. Double-blind comparison of optimal doses. Chest 1980;78:816-8.

3 Dullinger D, Dronenberg R, Niewoehner DE. Efficacy of inhaled metaproterenol and orally-administered theophylline in patients with chronic airflow obstruction. Chest 1986;89:171-3.

4 Dull W, Alexander $M$. Theophylline in stable chronic airflow obstruction. A reappraisal. Arch Intern Med 1984; 114:2399-401

5 Rogers RM, Owens GR, Pennock BE. The pendulum swings again. Toward a rational use of theophylline. Chest 1985;84:280-2.

6 Au WY, Dutt AK, DeSoyza N. Theophylline kinetics in chronic obstructive airways disease in the elderly. Clin Phronic obstructive airways diseal Ther 1985;37:472-8.

7 Guyatt GH, Townsend $M$, Pugsley SO, et al. Bronchodilators in chronic airflow limitation. Effects on airway function, exercise capacity and quality of life. Am Rev Respir Dis 1987;135:1069-74.

8 Taylor DR, Buick B, Kinney C, Lowry RC, McDevitt DG The efficacy of orally administered theophylline, inhaled salbutamol and a combination of the two as chronic therapy in the management of chronic bronchitis with reversible air-flow obstruction. Am Rev Respir Dis 1985;131:747-51.

9 Corris PA, Neville E, Nariman S, Gibson GJ, Doseresponse study of inhaled salbutamol powder in chronic response study of inhaled salbutamol powder
airflow obstruction. Thorax 1983;38:292-6.

10 Dull WL, Alexander MR, Sadoul P, Woolson RF. The efficacy of isoproterenol inhalation for predicting the response to orally administered theophylline in chronic obstructive pulmonary disease. Am Rev Respir Dis 1982;126:656-9.

11 Anderson G, Peel ET, Pardoe T, Jones R. Sustained release theophylline in chronic bronchitis. $\mathrm{Br} \mathrm{J} \mathrm{Dis} \mathrm{Chest}$ 1982;76:261-5. 